\title{
DRUG-INDUCED EOSINOPHILIA SECONDARY TO THE COTRIMOXAZOLE ADMINISTRATION IN ACUTE BRUCELLOSIS
}

\author{
JALIL MOSHARI ${ }^{1}$, HAMID REZA NIAZKAR ${ }^{2}$, MEHDI KARIMI SHAHRI ${ }^{3}$, MOHAMMAD GHORBANI $^{4 *}$, \\ RAHELEH DARAFSHI ${ }^{5}$
}

${ }^{1}$ Department of Pediatrics, School of Medicine, Gonabad University of Medical Sciences, Gonabad, Iran. ${ }^{2}$ Student Research Committee, Gonabad University of Medical Sciences, Gonabad, Iran. ${ }^{3}$ Department of Clinical Sciences, Faculty of medicine, Gonabad University of Medical Sciences, Gonabad, Iran. ${ }^{4}$ Department of Hematology and Blood Banking, Gonabad University of Medical Sciences, Gonabad, Iran. ${ }^{5}$ Department of Pediatrics, Faculty of Medicine, Gonabad University of Medical Sciences, Gonabad, Iran. Email: Sbmu_mohamad@yahoo.com

Received: 03 March 2018, Revised and Accepted: 14 May 2018

ABSTRACT

Peripheral blood eosinophilia is a common finding in the daily clinical practice. Drug-induced eosinophilia is usually benign but sometimes can be accompanied by tissue damage. In most cases, the eosinophilia resolves once the drug is withdrawn. However, the disease in some cases can persist in spite of the drug withdrawal. In this study, a case of drug-induced eosinophilia secondary to the cotrimoxazole intake is presented.

Keywords: Cotrimoxazole, Eosinophilia, Brucellosis.

(C) 2018 The Authors. Published by Innovare Academic Sciences Pvt Ltd. This is an open access article under the CC BY license (http://creativecommons. org/licenses/by/4. 0/) DOI: http://dx.doi.org/10.22159/ajpcr.2018.v11i9.25610

\section{INTRODUCTION}

Peripheral blood eosinophilia is a common finding in the daily clinical practice and usually accompanied by a group of signs and symptoms, which can help us roll out the differential diagnosis [1,2]. Peripheral eosinophilia can be divided into three categories including primary, secondary, and idiopathic eosinophilia.

Primary eosinophilia is diagnosed when there is a clonal expansion of eosinophil's lineage such as acute leukemia or chronic myeloid disorder. Secondary eosinophilia is diagnosed when eosinophilia occurs as an immunological response to an offending agent, which may be infectious or non-infectious. The examples of secondary eosinophilia include infections, drugs, the pulmonary eosinophilia, miscellaneous, and other causes of autoimmune/inflammatory/toxic origin, malignancies such as metastatic cancer and Hodgkin lymphoma, and endocrine disorders such as Addison's disease or growth factor deficiency. Idiopathic eosinophilia is diagnosed when no signs of primary or secondary eosinophilia can be seen despite a thorough evaluation [3]

The upper limit of the normal number for the blood eosinophil varies since the eosinophil count depends on age, time of day, exercise, and environmental stimuli, particularly allergen exposure [4]. Blood eosinophil counts undergo diurnal variation and become the lowest in the morning and the highest at night. A value $>600 / \mathrm{cmm}^{3}$ is usually considered abnormal and called eosinophilia. It is categorized into mild (600-1500 cells/ml), moderate $(1500-5000$ cells/ml), or severe $(>5000$ cells $/ \mathrm{ml}$ ) [5]. Common causes of eosinophilia are listed in Table 1.

\section{CASE REPORT}

A 4-year-old boy was admitted to our hospital complaining of chronic ostealgia in lower limbs and pelvic region. His parents complained of his loss of appetite, aggressiveness, and impatience behavior. He had the pain for a week while he experienced an inconstant fever followed by sweating mostly during night times afterward. Physical examination revealed intramouth ulcers in the tonsil's fold and peripheral tenderness in long bones. Abdomen examination was normal with no organomegaly or tenderness. He has been playing with a kid recently. He has no history of unboiled milk consumption but had cheeses. Serology tests such as wright test confirmed brucellosis. After diagnosing the Brucellosis, we prescribed rifampin and cotrimoxazole treatment. During the followup, after 2 weeks, the patient developed a peripheral eosinophilia secondary to the cotrimoxazole administration. Peripheral eosinophilia resolved once we withdraw the cotrimoxazole intake. Complete blood count (CBC) and serology tests are listed in Table 2.

\section{DISCUSSION}

Drug-induced eosinophilia is usually benign but sometimes can be accompanied by tissue damage, as in hypersensitivity pneumonitis. Patients with persistent eosinophilia should have periodic clinical and echocardiographic examinations to be detected for eosinophil-mediated cardiac damage, no matter how benign the eosinophilia may seem [6]. Most of the times, the eosinophilia resolves once the drug is withdrawn. However, the disease can persist in spite of the drug withdrawal in some cases. End-organ damage occurs in case of moderate-to-severe eosinophilia persistent [6]. Since blood eosinophil numbers do not necessarily indicate the extent of eosinophil involved in the affected tissues, eosinophilic tissue infiltration and end-organ manifestations can occur in the absence of peripheral eosinophilia [5]. In such cases, measurement of eosinophil granule proteins in serum, sputum, or other body fluids can help to monitor the disease activity [7].

Due to the sulfamethoxazole substances, cotrimoxazole (TMP-SMZ) can induce eosinophilia, and it has been used to treat a variety of bacterial infections. In children younger than 8 years old, administration of rifampin and TMP-SMZ for 6 weeks is the therapy choice for brucellosis. Since the secondary eosinophilia can be occurred by a variety of causes, before any further clinical investigations, it is important to take into account the drug-induced eosinophilia to prevent unnecessary diagnostic tests. We presented a case of eosinophilia secondary to the cotrimoxazole intake. Cotrimoxazole is also one of the probable causes of DRESS syndrome [8,9].

Peripheral eosinophilia is one of the most important findings in the sulfonamide administration. According to some case reports, Peripheral eosinophilia secondary to the sulfonamide administration,could be 
Table 1: Common causes of Eosinophilia

\begin{tabular}{ll}
\hline Condition & Causes \\
\hline Parasitic conditions & Strongyloidiasis: Strongyloides stercoralis \\
& Liver fluke: Fasciola hepatica \\
& Hydatid disease: Echinococcus granulosus \\
Allergy/atopy & Schistosomiasis: Schistosoma haematobium, S. mansoni, S. japonicum \\
Reactive eosinophilia & Asthma, urticaria, eczema, allergic rhinitis, angioneurotic edema \\
Atopy and allergic drug reactions & T-cell lymphoma, B-cell, lymphoma, acute lymphoblastic leukemia, eosinophilic leukemia \\
Collagen vascular diseases & Aspirin, penicillin, cephalosporin, nitrofurantoin, sulfonamide, iodides \\
Skin diseases & Rheumatoid arthritis, eosinophilic fasciitis, allergic angiitis, sarcoidosis \\
Idiopathic hypereosinophilic syndrome & Pemphigus, dermatitis herpetiformis, erythema multiform \\
\hline
\end{tabular}

Table 2: Result of the CBC and serologic tests

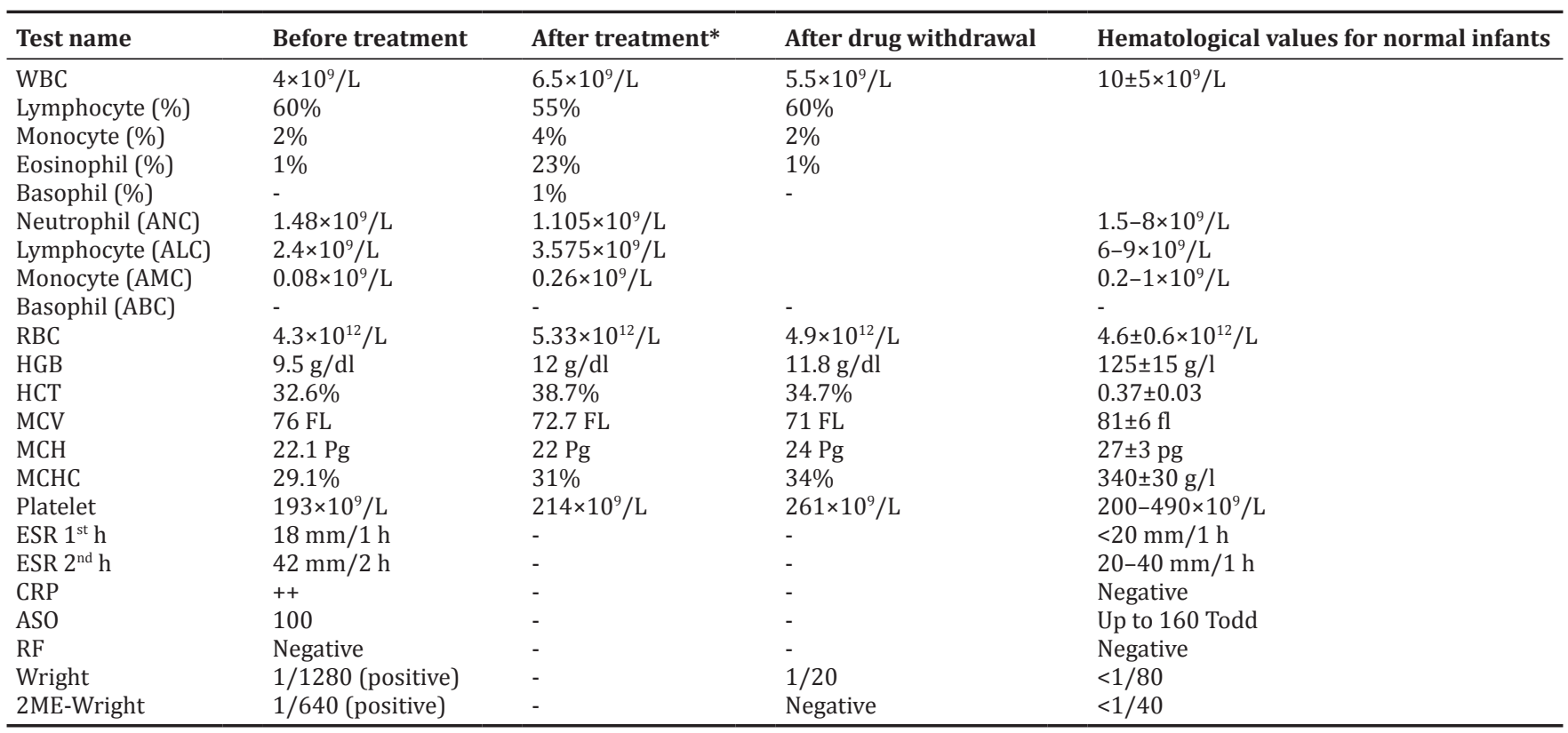

*These tests were performed after 2 weeks. CBC: Complete blood count, WBC: White blood cell, RBC: Red blood cell, MCV: Mean corpuscular volume, MCH: Mean corpuscular hemoglobin, CRP: C-reactive protein, ESR: Erythrocyte sedimentation rate, ASO: Anti-streptolysin O, HGB: Hemoglobin, HCT: Hematocrit, MCHC: Mean corpuscular hemoglobin concentration

a developing sign of migratory pneumonia or "atypical pneumonia" in case of chronic brucellosis [10]. Authors suggest asking for a CBC test before prescribing any kinds of sulfonamides to make sure the eosinophilia is induced by the drugs intake once the sulfonamides administration developed a peripheral eosinophilia.

\section{AUTHOR'S CONTRIBUTIONS}

JM and RD were the patient's doctors and MKS and MG followed up the patient and analyzed his laboratory tests. MG and HRN wrote the draft of the manuscript.

\section{REFERENCES}

1. Roufosse F, Weller PF. Practical approach to the patient with hypereosinophilia. J Allergy Clin Immunol 2010;126:39-44.

2. Lakshmi R, Liniya S, Vijayalakshmi S. Dapsone induced hypersensitivity syndrome - A case report. Int J Pharm Pharm Sci 2015;7:585-7.

3. Making AD. Approach to a case of eosinophilia. Ind J Aerosp Med
$2009 ; 53: 2$

4. Wardlaw A. Eosinophils in the 1990s: New perspectives on their role in health and disease. Postgrad Med J 1994;70:536-52.

5. Singh V, Gomez VV, Swamy SG, Vikas B. Approach to a case of eosinophilia. Int J Agile Syst Manage 2009;53:58-64.

6. Rothenberg ME. Eosinophilia. New Engl J Med 1998;338:1592-600.

7. Makiya MA, Herrick JA, Khoury P, Prussin CP, Nutman TB, Klion AD. Development of a suspension array assay in multiplex for the simultaneous measurement of serum levels of four eosinophil granule proteins. J Immunol Methods 2014;411:11-22.

8. Cacoub P, Musette P, Descamps V, Meyer O, Speirs C, Finzi L, et al. The dress syndrome: A literature review. Am J Med 2011;124:588-97.

9. Calaça GN, Pessoa CA, Wohnrath K, Nagata N. Simultaneous determination of sulfamethoxazole and trimethoprim in pharmaceutical formulations by square wave voltammetry. Int $\mathrm{J}$ Pharm Pharm Sci 2014;6:438-2.

10. Fiegenberg DS, Weiss H, Kirshman H. Migratory pneumonia with eosinophilia: Associated with sulfonamide administration. Arch Intern Med 1967;120:85-9. 